\title{
Application of solidified floating double-solvent dispersive liquid-phase microextraction for the analysis of the main active components in Zicao Chengqi decoction
}

\author{
Xiang Geng ${ }^{1,2} \cdot$ Xuan Chen ${ }^{1} \cdot{\text { Zhuoyi } \mathrm{Li}^{1} \cdot \text { Xiaohong Bai }}^{1} \cdot$ Shuang Hu${ }^{1}$
}

Received: 9 July 2020 / Accepted: 17 September 2020 / Published online: 26 September 2020

(c) Springer Nature Switzerland AG 2020

\begin{abstract}
A new method was established using solidified floating double-solvent dispersive liquid-phase microextraction combined with high-performance liquid chromatography (HPLC) for the simultaneous determination and extraction of six active ingredients from Aurantii Fructus Immaturus, Magnoliae Officinalis Cortex, Rhei Radix et Rhizoma, and Arnebiae Radix in the Zicao Chengqi decoction. The six active ingredients in Zicao Chengqi decoction include naringenin, honokiol, shikonin, magnolol, emodin, and $\beta, \beta$-dimethylacrylshikonin. In this experiment, the effects of the extractant type and volume, sample phase volume, sample phase $\mathrm{pH}$, extraction time, and stirring speed on the enrichment factor were studied, and optimal extraction conditions were determined. Naringenin $(6-600 \mathrm{ng} / \mathrm{mL})$, honokiol $(3-219 \mathrm{ng} / \mathrm{mL})$, shikonin (4-350 ng/mL), magnolol (3-230 ng/mL), emodin (3-190 ng/mL), and $\beta, \beta$-dimethylacrylshikonin (3-430 ng/mL) had a good linear relationship and all the correlation coefficients were 0.9900-0.9992. This method was used for the simultaneous concentration and enrichment of the six active ingredients from the Chinese herbal compound, Zicao Chengqi decoction, with recoveries between 95.7 and 100.3\%. The results showed that solidified floating double-solvent dispersive liquid-phase microextraction combined with HPLC can be used for the simultaneous extraction and analysis of multiple active components in the Zicao Chengqi decoction.
\end{abstract}

Keywords High-performance liquid chromatography · Solidified floating organic drop dispersive liquid-phase microextraction - Zicao chengqi decoction

\section{Introduction}

The Zicao Chengqi decoction, from Vol. 6 of the Zheng Zhi Zhun Sheng-you Ke, is prepared from four traditional Chinese herbs, including Aurantii Fructus Immaturus, Magnoliae Officinalis Cortex, Rhei Radix et Rhizoma, and Arnebiae Radix. It is a famous Chinese herbal medicine that relieves heat and removes toxins. At present, as a formula, the efficacy and side effects of the Zicao Chengqi decoction have not been systematically assessed, although some studies have investigated the efficacy of the active compound.[1]. According to previous studies, emodin in Rhei Radix et Rhizoma also displays antitumor, anti-inflammatory, and antibacterial activity [2-4]. Shikonin and $\beta, \beta-$ dimethylacrylshikonin in Arnebiae Radix have been shown to exhibit many biological and pharmacological activities,

Co-first author: Professor Xuan Chen.

Electronic supplementary material The online version of this article (https://doi.org/10.1007/s42452-020-03557-6) contains supplementary material, which is available to authorized users.

$\triangle$ Xuan Chen, ywfxcx@163.com | ${ }^{1}$ School of Pharmacy, Shanxi Medical University, Jinzhong 030619, China. ${ }^{2}$ Department of Pharmacy, Shanxi Health Vocational College, Jinzhong 030619, China. 
including antimicrobial, antioxidant, and, antitumor activities $[5,6]$. Magnolol and honokiol are two compounds with a high content in Magnoliae Officinalis Cortex; they have similar chemical structures and their activity is closely related to the traditional efficacy of Magnoliae Officinalis Cortex, which is an important pharmacodynamic material for antibacterial activity $[7,8]$. Flavone glycosides in Aurantii Fructus Immaturus have the functions of gastric emptying and promoting intestinal peristalsis, among which naringin has significant anti-inflammatory and analgesic effects $[9,10]$. Studies have shown that naringin and naringenin show essentially the same biological behavior. Naringin cannot be detected in plasma since it is deglycosylated by intestinal bacteria to produce naringenin, which is then absorbed into the liver. Therefore, naringenin content can be measured to determine the naringin content [11-13]. Thus, naringenin, honokiol, shikonin, magnolol, emodin, and $\beta, \beta$-dimethylacrylshikonin were selected as active components in this study. The properties of the active components in this preparation are significantly different; however, these components all at low concentrations and exist in the same complex sample matrix [1]. Therefore, it is of considerable importance to establish a rapidly and highly repeatable sample pretreatment technique for the simultaneous extraction and concentration of low concentration target analytes in Chinese herbal compound preparations.

Liquid-phase microextraction (LPME) [14], solid-phase microextraction (SPME) [15], and other miniaturized sample preparation technologies are widely used for the enrichment and concentration of trace compounds in various complex samples. Compare with recent microextraction techniques for determination of organic and inorganic compounds such as microextraction based on deep eutectic solvent [16-18], continuous sample drop flow microextraction [19], dispersive liquid-liquid microextraction based on solidification of floating organic drop [20-22] and solid phase extraction combined with dispersive liquid-liquid microextraction [23], LPME and SPME have the advantages of simple operation, minimal organic solvent use, low cost, low environmental impact, and high enrichment factor $(E F)$; thus, they are suitable for modern drug analysis. In 2007, Khalili-Zanjani et al. [24] proposed a new LPME method i.e., solidification of a floating organic drop liquid-phase microextraction (SFOD-LPME), for the pre-enrichment of polyaromatic hydrocarbons in well-water samples. The melting point (MP) of the extractant in SFOD-LPME should be close to room temperature $\left(10-30{ }^{\circ} \mathrm{C}\right)$ and the density must be lower than that of water; thus, in traditional SFODLPME, n-undecane $\left(M P=26{ }^{\circ} \mathrm{C}\right.$, density $\left.=0.74 \mathrm{~g} / \mathrm{cm}^{3}\right)$, n-dodecane $\left(\mathrm{MP}=9.6^{\circ} \mathrm{C}\right.$, density $\left.=0.75 \mathrm{~g} / \mathrm{cm}^{3}\right)$, 1-undecanol $\left(\mathrm{MP}=19^{\circ} \mathrm{C}\right.$, density $\left.=0.83 \mathrm{~g} / \mathrm{cm}^{3}\right)$, and 1-dodecanol
$\left(\mathrm{MP}=24{ }^{\circ} \mathrm{C}\right.$, density $\left.=0.83 \mathrm{~g} / \mathrm{cm}^{3}\right)$ are usually used as the extracting agents. When the extraction solvent is quickly added to the sample solution under agitation, it immediately forms a turbid suspension and many organic droplets are evenly dispersed in the solution. These droplets have a large surface area, and therefore, a large contact area with the sample solution, which accelerates the extraction balance and increases the enrichment coefficient in trace target analytes. SFOD-LPME has been applied to the extraction and preconcentration of amantadine [25], the extraction of pyrethroid insecticides in fresh fruits and fruit juices [26], the application in determination of some pesticides in milk samples [27], the determination of phenolic endocrine disrupting chemicals in bottled beverages [28], the extraction of carbamate pesticides from fruit juice and vegetable samples [29], the determination of triazole fungicides in water samples [30], and the extraction and depuration of pantoprazole in rat plasma [31].

In our previous study, we adopted SFOD-LPME for up to $468 \mathrm{EFs}$ in 6 target analytes in the water matrixes. This microextraction technique combined with HPLC and other detection techniques. The operation is simple and cheap, and it is easy to obtain a high enrichment ratio. In addition, there have be no relevant reports of SFOD-LPME methods for simultaneous extraction of all these six active ingredients in the Zicao Chengqi decoction. Therefore, in this study, SFOD-LPME was used to simultaneously concentrate and enrich the active ingredients in the extract of Zicao Chengqi decoction.

\section{Methods and materials}

\subsection{Materials and instruments}

\subsubsection{Reagent}

Magnolol (batch number: MUST-18032102, purity: 99.71\%), honokiol (batch number: MUST-18032205, purity: 99.34\%), shikonin (batch number: MUST-18040409, purity: 98.84\%), emodin (batch number: MUST-1710270, purity: 99.48\%), $\beta, \beta$-dimethylacrylshikonin (batch number: MUST17101201, purity: $98.60 \%$ ), and naringenin (batch number: MUST-18032406, purity: 99.18\%) were purchased from Manster Biotechnology Co., Ltd. (Chengdu, China). 1-Undecanol, 1-dodecanol, hexadecane, and glacial acetic acid (analytically pure) were purchased from Tianjin Guangfu Fine Chemical Research Institute (Tianjin, China). Aurantii Fructus Immaturus, Magnoliae Officinalis Cortex, Rhei Radix et Rhizoma and Arnebiae Radix were purchased from Beijing Tong Ren Tang Pharmacy (Taiyuan, China). HPLC-grade methanol was obtained from Tianjin Siyou Chemical (Tianjin, China). Hydrochloric acid $(\mathrm{HCl})$ was 
purchased from Beijing Chemical Works (Beijing, China). The water used in the experiment was double distilled.

\subsubsection{Preparation of standard solution}

Six traditional Chinese medicine standard substances were used. The standard solution of traditional Chinese medicine contained $0.24 \mathrm{mg}$ of naringenin, $0.23 \mathrm{mg}$ of honokiol, $0.6 \mathrm{mg}$ of shikonin, $0.23 \mathrm{mg}$ of magnolol, $0.2 \mathrm{mg}$ of emodin, and $0.76 \mathrm{mg}$ of $\beta, \beta$-dimethylacrylshikonin per $1 \mathrm{~mL}$, and was prepared with methanol. This was stored at $4{ }^{\circ} \mathrm{C}$. Before use, the six standard solutions were mixed in a certain proportion to make a mixed standard solution with double-distilled water.

\subsubsection{Preparation of Zicao Chengqi decoction}

The powder of the four herbs (Aurantii Fructus Immaturus, Magnoliae Officinalis Cortex, Rhei Radix et Rhizoma, and Arnebiae Radix) was weighed and mixed according to the compound ratio of Zicao Chengqi decoction (1:2:4:1). This was placed in a conical bottle with a plug and $25 \mathrm{~mL}$ of methanol was added. After shaking, the bottle was closed, the content was weighed, and it was extracted with methanol by ultrasonication for $45 \mathrm{~min}$. This was left to cool at room temperature. The lost weight was replaced with methanol and the mixture was shaken and centrifuged under $2500 \mathrm{rpm}$ for $4 \mathrm{~min}$. The supernatant was stored at $4{ }^{\circ} \mathrm{C}$ for later use. Before the experiment, an appropriate amount of supernatant was extracted and diluted 50 times with double-distilled water.

\subsubsection{Preparation of sample solution}

A $100 \mu \mathrm{L}$ sample of the mixed reference or testing solution was diluted to $7 \mathrm{~mL}$ of sample solution at specific concentrations; the sample solution was adjusted to $\mathrm{pH} 4$ with $0.1 \mathrm{~mol} / \mathrm{L} \mathrm{HCl}$.

\subsection{Experimental method}

\subsubsection{Chromatographic condition}

A Shimazu LC-20AT HPLC. C18 column $(250 \times 4.6 \mathrm{~mm}, 5 \mu \mathrm{m}$; Agilent, Eclipse Plus, Santa Clara, CA, USA) (wavelength $294 \mathrm{~nm}$, column temperature $30^{\circ} \mathrm{C}$, flow velocity $1 \mathrm{~mL} /$ min, injection volume $20 \mu \mathrm{L}$ ). The mobile phase was the gradient elution of methanol (A) to $0.2 \%$ glacial acetic acid solution (B). The elution procedure was as follows: $40 \%$ B (0-6 min), 40\%-23\% B (6-10 min), 23\% B (10-11 min), $23 \%-20 \%$ B (11-25 min), 20\%-5\% B (25-27 min), and $5 \%-40 \%$ B (27-37 min).

\subsubsection{Instrument and equipment}

A Shimazu LC-20AT HPLC, Shimazu CTO-10AS type column thermostat, Shimazu SPD-20A UV-VIS detector, 85-2 digital display constant temperature magnetic stirrer (Jiangsu Jintan Ronghua Instrument Manufacturing Co., Ltd), SI-114 electronic balance (Shanghai Liheng Instrument Co., Ltd), and KQ 3200 DB CNC ultrasonic cleaner (Kunshan Ultrasonic Instrument Co., Ltd) were used.

\subsubsection{Experimental procedure}

A glass vial that contained $7 \mathrm{~mL}$ of sample solution was fixed on the magnetic stirrer (Fig. 1). Double extractants of 1-undecanol-1-dodecanol $(20 \mu \mathrm{L}, 3: 7, \mathrm{v} / \mathrm{v})$ were added to the sample solution. The magnetic stirrer was turned on and the extraction was performed at $1200 \mathrm{rpm}$ for $30 \mathrm{~min}$. After extraction, the sample bottle was left for $5 \mathrm{~min}$. The droplets of double extractants were suspended on the surface of the solution and the sample bottle was slowly rotated. After the suspension droplets gathered, the sample bottle was immediately placed in the refrigerator $\left(-20^{\circ} \mathrm{C}\right)$ and was frozen for $6 \mathrm{~min}$. The solidified aggregates were removed with a small spoon, placed in a $500 \mu \mathrm{L}$ centrifuge tube, dissolved at room temperature, diluted, and mixed with $20 \mu \mathrm{L}$ of methanol; $20 \mu \mathrm{L}$ of the solution was used for HPLC analysis.

\section{Results and discussion}

\subsection{Optimization of extraction conditions}

\subsubsection{Proportion of double extractants}

In this experiment, the extractant should meet the following requirements: MP close to room temperature $\left(10-30^{\circ} \mathrm{C}\right)$; insoluble in water, which can provide suitable extraction efficiency and high concentration factor; and less dense than water. Thus, we chose three extractants, 1-undecanol, 1-dodecanol, and hexadecane. When one extractant was used alone, the EFs of the six analytes in hexadecane were lower than those in the other two extractants. The other two extractants showed no significant difference in EFs in the six analytes. In 1-dodecanol, the $E F$ of $\beta, \beta$-dimethylacrylshikonin was low because the poor peak shape of $\beta, \beta$-dimethylacrylshikonin could not be accurately integrated in the chromatogram. However, in 1 -undecanol, the peak shape of $\beta, \beta$-dimethylacrylshikonin was significantly better than that of 1-dodecanol; thus, its $E F$ was significantly higher, but the $E F$ of emodin was lower. Thus, the double-solvent model of 1-undecanol and 1-dodecanol was considered useful. The effects of 


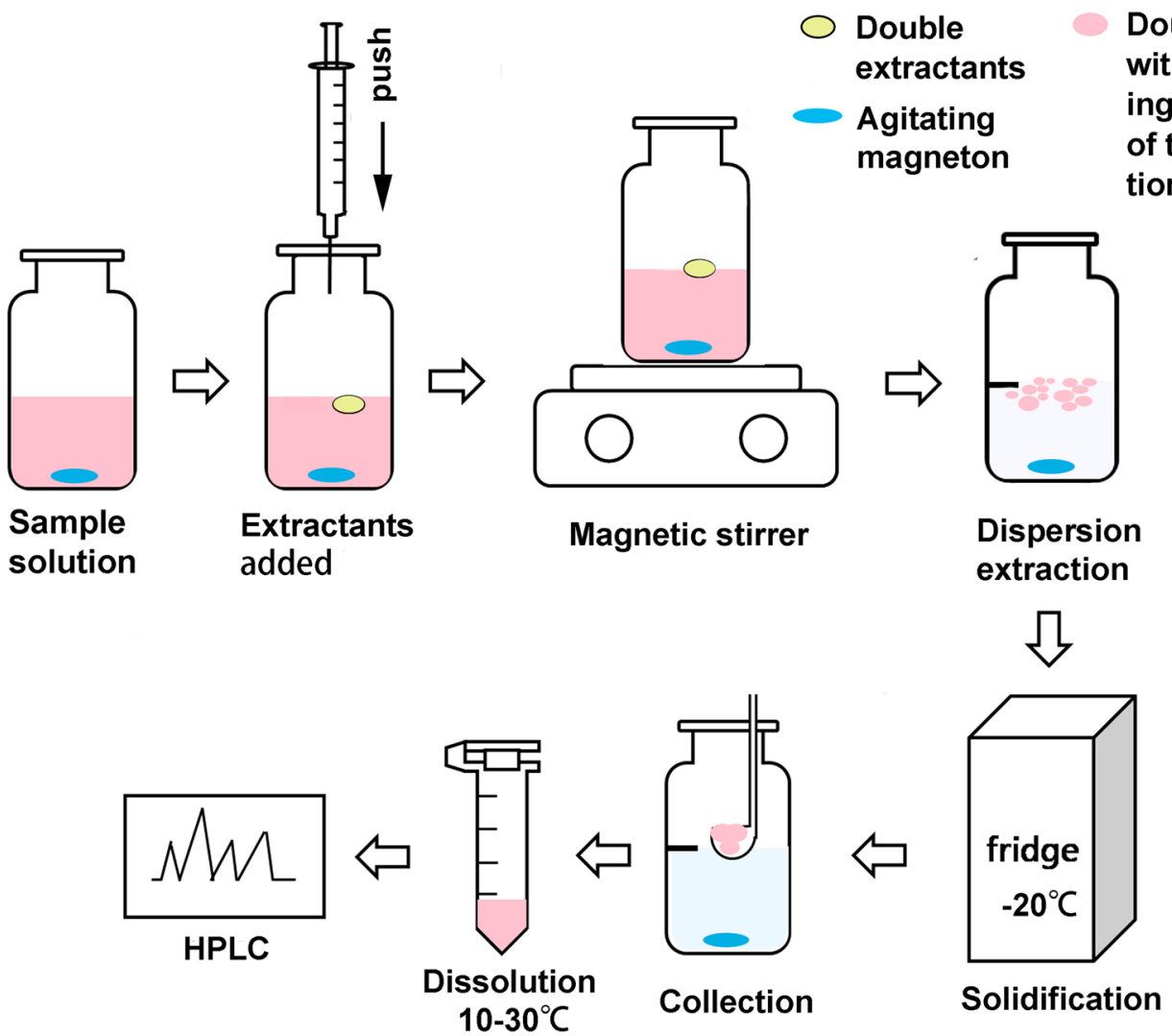

Fig. 1 A simplified schematic diagram of SFOD-DS-DLPME-HPLC
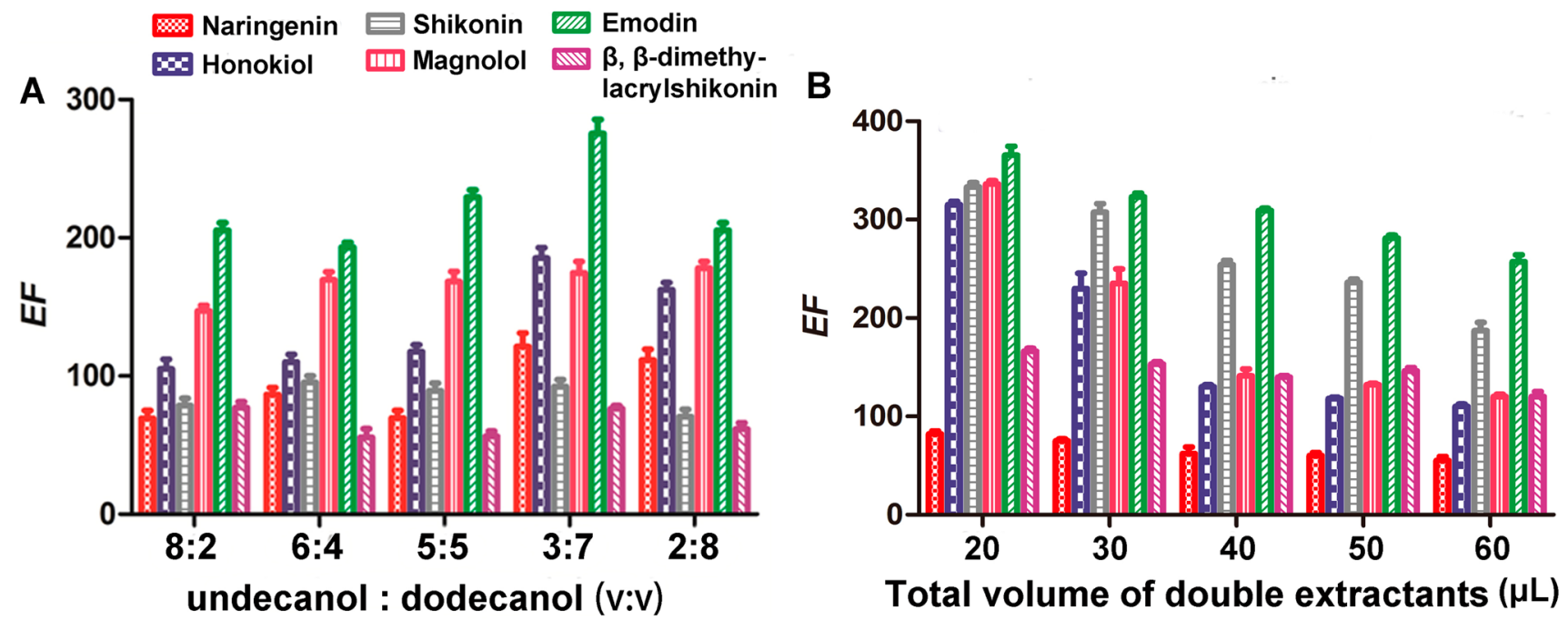

Fig. 2 Selection of the proportion and total volume of double extractants. The effect of volume ratio (a) and total volume (b) of the double extractants on the EFs of the target analytes 
the double extractants at different volume ratios $(8: 2,6: 4$, $5: 5,3: 7$, and 2:8) on the EFs of the target analytes were investigated in this study. The $E F$ and peak shape of the six target analytes were best when the ratio of 1-undecanol and 1-dodecanol was 3:7 (Fig. 2a).

\subsubsection{Total volume of double extractants}

A 3:7 volume ratio of 1-undecanol:1-dodecanol was used as the double extractant. The total volume of the double extractant greatly affected the EFs of the target analytes. An excessively large volume was not conducive to the aggregation and collection of the dispersion solutions. The effect of the total volume of double extractants (20, $30,40,50$, and $60 \mu \mathrm{L}$ ) on $E F s$ in six target analytes were investigated. When the total volume of the double extractants was $20 \mu \mathrm{L}$, the EFs in the six target analytes were the largest (Fig. 2b). Therefore, $20 \mu \mathrm{L}$ was selected as the total volume of the double extractants.

\subsubsection{Sample phase $\mathrm{pH}$}

The $\mathrm{pH}$ will affect the form of the target analytes in the sample solution, so the $\mathrm{pH}$ will play an important role in the extraction efficiency. In this experiment, all the six target analytes have a certain weak acidity. When the $\mathrm{pH}$ of the sample phase solution is acidic, it is advantageous for the target analyte to exist in the molecular state and thus be extracted by the organic solvent. It has been verified by experiments that when the sample phase is at $\mathrm{pH} 4$, the extraction efficiency of the target analyte is the highest. Therefore, the final choice is to adjust the $\mathrm{pH}$ of the sample phase to be 4 . The EFs in the six target analytes were largest when the $\mathrm{pH}$ of the sample phase was 4 (Fig. 3a). Therefore, $\mathrm{pH} 4$ was selected as the $\mathrm{pH}$ of the sample phase.
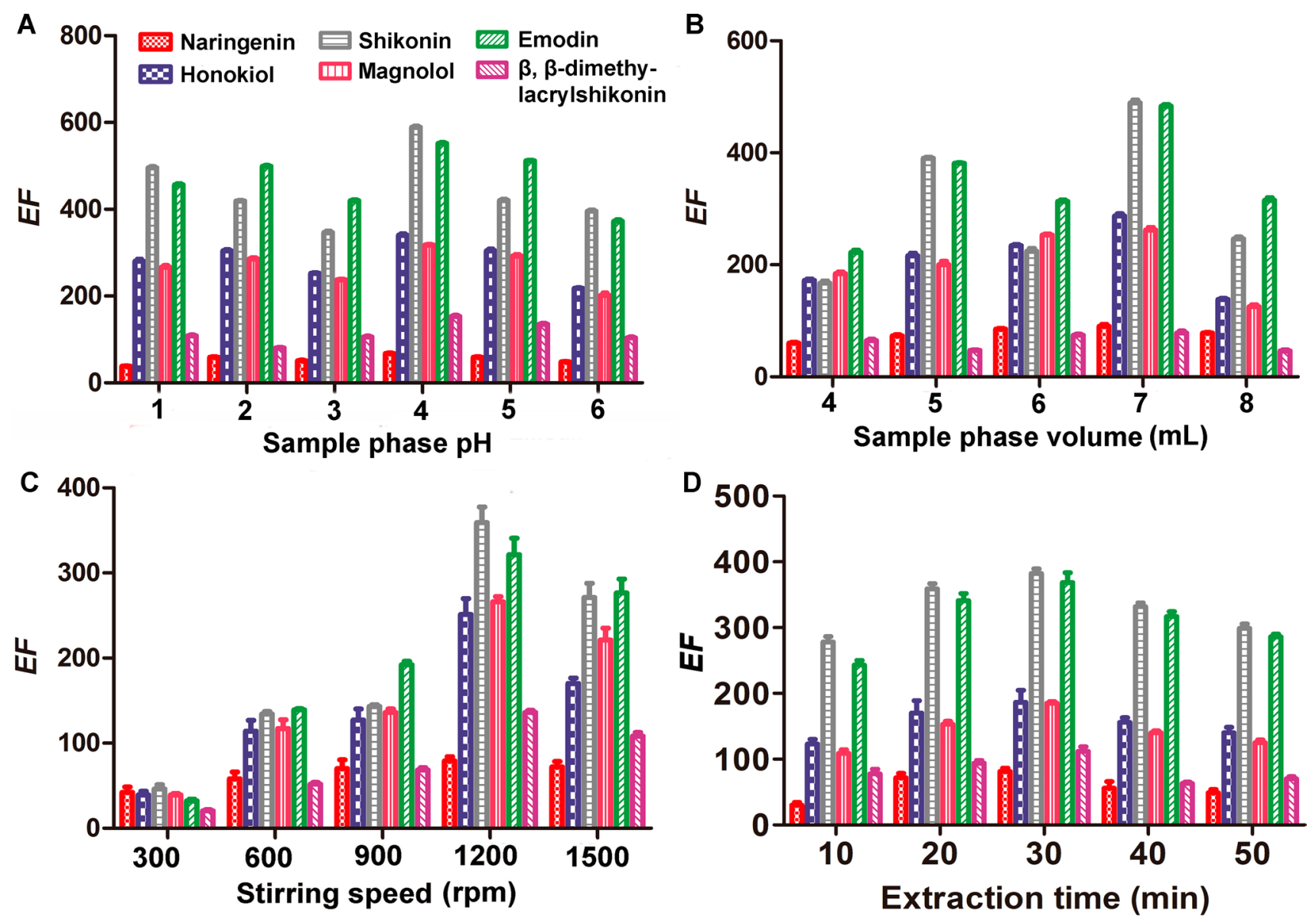

Fig. 3 Optimization of the extraction conditions. The effect of the sample phase pH (a), the sample phase volume (b), stirring speed (c), and extraction time (d) on the EFs of the target analytes 


\subsubsection{Sample phase volume}

The effect of different sample phase volumes $(4,5,6,7$, and $8 \mathrm{~mL}$ ) on the $E F \mathrm{~s}$ in six target analytes were investigated. When the sample phase volume was $7 \mathrm{~mL}$, the $E F s$ in the six target analytes were largest (Fig. 3b). Therefore, $7 \mathrm{~mL}$ was selected as the sample phase volume.

\subsubsection{Stirring speed}

Stirring can accelerate mass transfer and promote the dispersion of the sample solution; thus, stirring improves the extraction efficiency of the analyte in the extraction phase. An increase in stirring speed can improve $E F$; however, if the extraction speed is too high, the solution will spatter, and organic droplets will be destroyed. Different stirring speeds $(300,600,900,1200$, and $1500 \mathrm{rpm})$ were investigated. The stirring speed of $1200 \mathrm{rpm}$ obtained the highest EFs (Fig. 3c). Therefore, $1200 \mathrm{rpm}$ was selected as the stirring speed.

\subsubsection{Extraction time}

Extraction time is also an important parameter to improve the $E F s$ of target analytes. In order to accelerate the extraction process, extraction time was also evaluated by optimizing the duration of the agitation process. The effect of extraction time $(10,20,30,40$, and $50 \mathrm{~min})$ on the $E F \mathrm{~s}$ in six target analytes were investigated. The highest $E F$ s were obtained with high enrichment efficiency for all six target analytes at $30 \mathrm{~min}$. Therefore, $30 \mathrm{~min}$ was selected as the extraction time (Fig. 3d).

\subsection{Method validation}

Under the optimal experimental conditions, several tests were validated, including linearities, LODs, LOQs, precisions of intraday and interday, and recoveries of the target analytes.
Table 2 The contents of the six target analytes in the Chinese herbal compound prescription of Zicao Chengqi decoction $(n=3)$

\begin{tabular}{|c|c|c|c|c|}
\hline \multirow[t]{2}{*}{ Analytes } & \multicolumn{3}{|c|}{ Analyte content $(\mu \mathrm{g} / \mathrm{g})$} & \multirow{2}{*}{$\begin{array}{l}\text { Average } \\
\text { content } \\
(\mu \mathrm{g} / \mathrm{g})\end{array}$} \\
\hline & 1 & II & III & \\
\hline Naringenin & 39.5 & 43.6 & 47.1 & 43.4 \\
\hline Honokiol & 42.6 & 43.0 & 44.0 & 43.2 \\
\hline Shikonin & 36.9 & 38.1 & 34.4 & 36.5 \\
\hline Magnolol & 96.5 & 98.5 & 87.7 & 94.2 \\
\hline Emodin & 20.9 & 20.6 & 19.3 & 20.3 \\
\hline$\beta, \beta$-Dimethylacrylshikonin & 74.3 & 73.0 & 80.8 & 76.0 \\
\hline
\end{tabular}

\subsubsection{Linearity, limit of detection (LOQ) and limit of quantification (LOD)}

The calibration curves were obtained by plotting peak areas $(y)$ against the concentrations of the six analytes $(x)$ in triplicate. $L O D$ and $L O Q$ were calculated at $\mathrm{S} / \mathrm{N}=3$ and 10, respectively. These results are shown in Table 1.

\subsubsection{Precision test}

The precision of the method is expressed by the relative standard deviation (RSD). A sample of the mixed reference solution of the six target analytes was precisely measured and prepared into mixed standard solutions at high, medium, and low concentrations. Intraday and interday precisions were measured by three replicate experiments of the standard solutions at high, medium, and low concentrations within 1 day, and with three replicates over 3 days (Table S1.)

\subsubsection{Recovery test}

The percentage recoveries of spiked samples were obtained by five replicate experiments. The appropriate amount of Zicao Chengqi decoction sample solution and mixed standard reserve solution were precisely

Table 1 Methodology parameters of the six target analytes

\begin{tabular}{|c|c|c|c|c|c|c|}
\hline Analytes & Regression equation & $\begin{array}{l}\text { Correlation } \\
\text { coefficient }\end{array}$ & Linear Range ( $\mu \mathrm{g} / \mathrm{mL})$ & $\mathrm{LOD}(\mathrm{ng} / \mathrm{mL})$ & LOQ (ng/mL) & $E F$ \\
\hline Naringenin & $Y=13379 X-16239$ & 0.9962 & $0.006-0.600$ & 1.5 & 6.0 & 83 \\
\hline Honokiol & $Y=5338.6 X+33,753$ & 0.9958 & $0.003-0.219$ & 0.6 & 3.0 & 252 \\
\hline Shikonin & $Y=1153 X+16,071$ & 0.9943 & $0.004-0.350$ & 0.4 & 2.0 & 468 \\
\hline Magnolol & $Y=3357.1 X+27,646$ & 0.9992 & $0.003-0.230$ & 0.5 & 3.0 & 229 \\
\hline Emodin & $Y=12418 X+109,642$ & 0.9972 & $0.003-0.190$ & 0.2 & 2.0 & 436 \\
\hline$\beta, \beta$-dimethylacrylshikonin & $Y=1259.1 X-8407.7$ & 0.9900 & $0.003-0.430$ & 0.6 & 2.4 & 92 \\
\hline
\end{tabular}

${ }^{*} E F=C_{\mathrm{e}} / C_{0}$, the $C_{\mathrm{e}}$ was obtained by the concentration of the analyte in the extraction phase after extraction and $C_{0}$ is obtained by that in the sample phase before extraction 


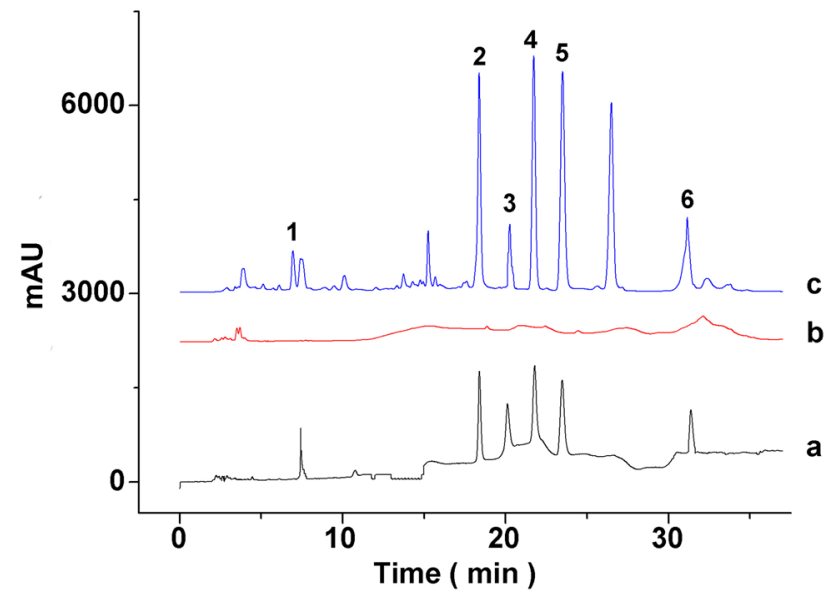

Fig. 4 The chromatogram of six active ingredients in the Chinese herbal compound prescription of the Zicao Chengqi decoction. 1: Naringenin; 2: Honokiol; 3: Shikonin; 4: Magnolol; 5: Emodin; 6: $\beta, \beta-$ dimethylacrylshikonin. a: reference substance; $b$ : before microextraction; and c: after microextraction

measured and the operation was performed according to the method in Sect. 2.2.3 (Table S2).

\subsection{Application to the testing sample}

SF-DS-DLPME combined with HPLC was used to determine the content in the six target analytes in the Chinese herbal compound prescription, Zicao Chengqi decoction. The results are shown in Table 2 . The chromatogram (Fig. 4) shows the excellent enrichment and separation of the active compounds by SF-DS-DLPME. SF-DS-DLPME can be used for the simultaneous enrichment, concentration, and determination of the main active material in Zicao Chengqi decoction.

\section{Microextraction mechanism}

In SF-DS-DLPME, two long-chain n-alcohols were first mixed in proportion to form a two-solvent supramolecular system. When this two-solvent system entered the sample phase, at a certain stirring speed, it was dispersed into a large number of tiny droplets and the supramolecular structure was rearranged to form a new supramolecular aggregate. This substantially increased the contact surface area between the analytes and the supramolecular aggregates, which was conducive to the extraction of analytes with different polarity through intermolecular hydrogen bonding or hydrophobic interaction, thus increasing the EFs. As the agitation stopped, the supramolecular molecules with the analytes were aggregated and floated on the surface of the sample phase, where they were solidified into spheres at low temperatures, and eventually collected. Therefore, this two-solvent supramolecular system realized the microextraction of analytes in the process of self-assembly.

\section{Comparison of the proposed method with the others}

To further clarify the feasibility of SF-DS-DLPME, we compared it with the other related analysis methods reported [32] (Table 3). As can be seen from Table 3, the EFs of analytes in SF-DS-DLPME are 3-14 times higher than those of the other methods; the extraction time is shortened by $15 \mathrm{~min}$ on average. Therefore, SF-DSDLPME has the following advantages: (1) it is simple and quick to operate, requiring no other auxiliary materials or special preparation; and (2) it relies on the self-assembly of the supramolecular system with double extractants to realize the simultaneous extraction of various active ingredients with different structures. Therefore, SF-DSDLPME is more suitable for the extraction and enrichment of the six active ingredients in the Zicao Chengqi decoction than these three alternative methods.

\section{Concluding remarks}

The active ingredients in Zicao Chengqi decoction were extracted, enriched, and determined by SFOD-LPME combined with HPLC in this study. The target analyte and extractant formed ordered, solid supramolecular aggregates by using mixed solvent instead of single solvent in this method, which greatly improved the separation and enrichment ratio of the target analyte. In conclusion, SFOD-LPME with undecanol-dodecanol mixed solvent as an extractant combined with HPLC can be used for simultaneous extraction and analysis of multiple active components in preparations of traditional Chinese medicine. 


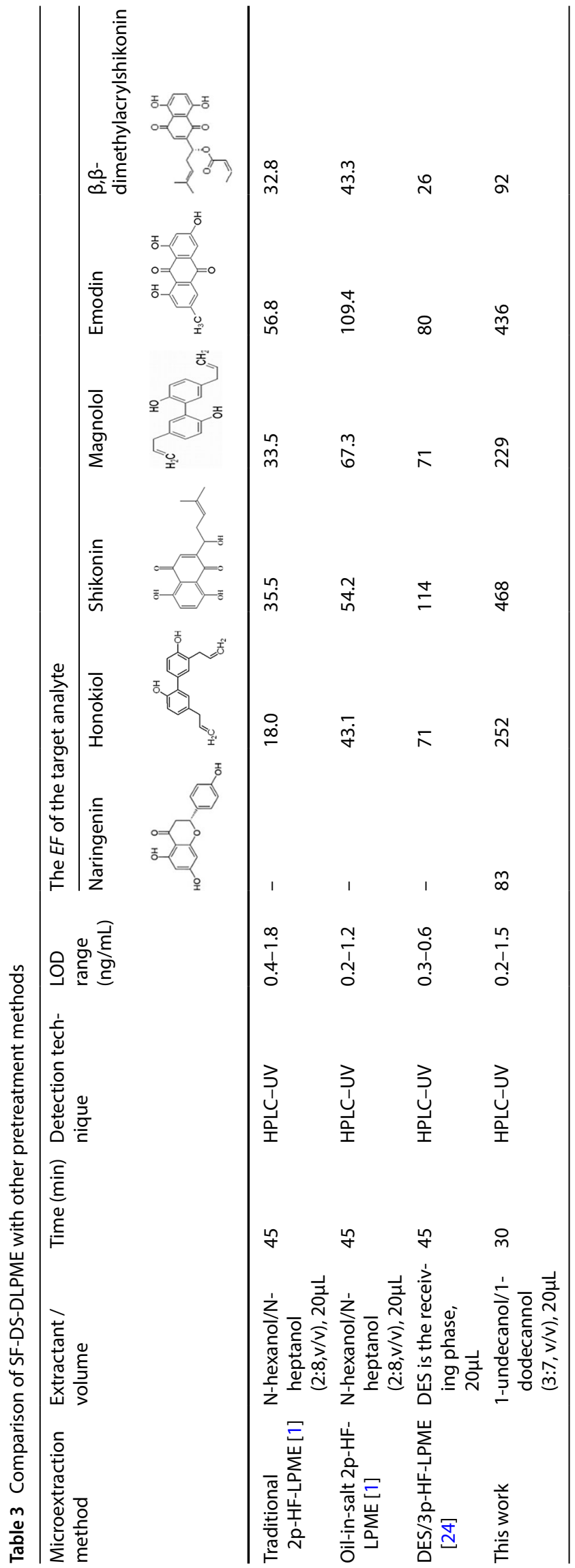

Funding This study was funded by the Natural Science Foundation of Shanxi (No. 201901D111207) and the National Natural Science Foundation of China (No. 81973287).

\section{Compliance with ethical standards}

Conflict of interest All authors declare that they have no conflicts of interest.

Ethical approval This article does not contain any studies with human participants or animals performed by any of the authors.

\section{References}

1. Li MM, Hu S, Chen X, Bai XH (2017) Development of a novel hollow-fiber liquid-phase microextraction based on oil-in-salt and its comparison with conventional one. J Sep Sci 40:2941-2949

2. Wang CG, Yang JQ, Liu BZ, Jin DT, Wang C, Zhong L, Zhu D, Wu $Y$ (2010) Anti-tumor activity of emodin against human chronic myelocytic leukemia K562 cell lines in vitro and in vivo. Eur J Pharmacol 627:33-41

3. Park SY, Jin ML, Ko MJ, Park G, Choi YW (2016) Anti-neuroinflammatory effect of emodin in LPS-stimulated microglia: involvement of AMPK/Nrf2 activation. Neurochem Res 41:2981-2992

4. Li L, Song X, Yin Z, Jia R, Li Z, Zhou X, Zou Y, Li L, Yin L, Yue G, Ye G, Lv C, Shi W, Fu Y (2016) The antibacterial activity and action mechanism of emodin from Polygonum cuspidatum against Haemophilus parasuis in vitro. Microbiol Res 186-187:139-145

5. Andújar I, Ríos JL, Giner RM, Recio MC (2013) Pharmacological properties of shikonin-a review of literature since 2002. Planta Med 79:1685-1697

6. Zhang LL, Zhan L, Jin YD, Min ZL, Wei C, Wang Q, Chen YJ, Wu QM, Hu XM, Yuan Q (2017) SIRT2 mediated antitumor effects of shikonin on metastatic colorectal cancer. Eur J Pharmacol 797:1-8

7. Park J, Lee J, Jung E, Park Y, Kim K, Park B, Jung K, Park E, Kim J, Park D (2004) In vitro antibacterial and anti-inflammatory effects of honokiol and magnolol against Propionibacterium sp. Eur J Pharmacol 496:189-195

8. Zhou G, Zhang WH, Mao QG, Wang YW (2019) Content and efficacy of six penylpropanoids in magnoliae officinalis cortex. $J$ Wuhan Univ (Nat Sci Ed) 65:340-346

9. Chen KH, Weng MS, Lin JK (2007) Tangeretin suppresses IL-1 beta-induced cyclooxygenase (COX)-2 expression through inhibition of p38 MAPK, JNK, and AKT activation in human lung carcinoma cells. Biochem Pharmacol 73:215-227

10. Xie RF, Wen SL, Li Y, Zuo C, Zhang JS (2011) Study on the antiinflammation and analgesia of naringin. J. Hunan Normal Univ (Med Sci) 8:5-9

11. Wang XJ, Chen X, Cao HX, Sun H, Yang K (2007) Pharmacokinetic studies of naringenin and hesperetin in human plasma after oral administration of Zhizhu Wan. Chin J Exp Trad Med Formulae 13:18-20

12. Wang $X J$, Chen $X$, Yang $K$, Ying JCL, Cao HX (2007) A qualitative and quantitative analysis: migrating constituents of Zhizhu pills in rat blood. World Sci Technol Mod Trad Chin Med Mat Med 9:54-56

13. Ma XQ, Li C, Yuan LH, Wang SJ, Liu XQ (2013) Pharmacokinetics of naringin and neohesperidin in the total flavanones of Zhishi extract in rats. J China Pharm Univ 44:161-166 
14. Yan YY, Chen X, Hu S, Bai XH (2014) Applications of liquid-phase microextraction techniques in natural product analysis: a review. J Chromatogr A 1368:1-17

15. Ma JP, Wang HW, Guan YF (2002) New technologies of solidphase microextraction. Chin J Chromatogr 20:16-20

16. Pirsaheb M, Fattahi N (2018) Development of a liquid-phase microextraction based on the freezing of a deep eutectic solvent followed by HPLC-UV for sensitive determination of common pesticides in environmental water samples. RSC Adv 21:11412-11418

17. Akramipour R, Golpayegani MR, Gheini S, Fattahi N (2018) Speciation of organic/inorganic mercury and total mercury in blood samples using vortex assisted dispersive liquid-liquid microextraction based on the freezing of deep eutectic solvent followed by GFAAS. Talanta 186:17-23

18. Habibollahi MH, Karimyan $K$, Arfaeinia $H$, Mirzaei N, Safari $Y$, Akramipour R, Sharafi H, Fattahi N (2019) Extraction and determination of heavy metals in soil and vegetables irrigated with treated municipal wastewater using new mode of dispersive liquid-liquid microextraction based on the solidified deep eutectic solvent followed by GFAAS. J Sci Food Agric 99:656-665

19. Karimaei M, Sharafi K, Moradi M, Ghaffari HR, Biglari H, Arfaeinia $\mathrm{H}$, Fattahi N (2017) Optimization of a methodology for simultaneous determination of twelve chlorophenols in environmental water samples using: in situ derivatization and continuous sample drop flow microextraction combined with gas chromatography-electron-capture detection. Anal Methods 19:2865-2872

20. Pirsaheb M, Fattahi N, Shamsipur M, Khodadadi T (2013) Application of dispersive liquid-liquid microextraction based on solidification of floating organic drop for simultaneous determination of alachlor and atrazine in aqueous samples. J Sep Sci 36:684-689

21. Ataee M, Ahmadi-Jouibari T, Fattahi N (2016) Application of microwave-assisted dispersive liquid-liquid microextraction and graphite furnace atomic absorption spectrometry for ultratrace determination of lead and cadmium in cereals and agricultural products. Int J Environ Anal Chem 96:271-283

22. Rezaee M, Khalilian F, Mashayekhi HA, Fattahi N (2014) A novel method for the high preconcentration of trace amounts of the aflatoxins in pistachios by dispersive liquid-liquid microextraction after solid-phase extraction. Anal Methods 10:3456-3461

23. Sadeghi $M$, Nematifar Z, Irandoust $M$, Fattahi $N$, Hamzei $P$, Barati A, Ramezani M, Shamsipur M (2015) Efficient and selective extraction and determination of ultra trace amounts of $\mathrm{Hg}^{2+}$ using solid phase extraction combined with ion pair based surfactant-assisted dispersive liquid-liquid microextraction. RSC Adv 5(122):100511-100521

24. Khalili Zanjani MR, Yamini Y, Shariati S, Jönsson JA (2007) A new liquid-phase microextraction method based on solidification of floating organic drop. Anal Chim Acta 585:286-293
25. Sorouraddin SM, Farajzadeh MA, Hassanyani A, Afshar Mogaddam MR (2016) Combination of homogenous liquid-liquid extraction and dispersive liquid-liquid microextraction for extraction and preconcentration of amantadine from biological samples followed by its indirect determination by flame atomic absorption spectrometry. RSC Adv 110:108603-108610

26. Torbati M, Farajzadeh MA, Torbati M, Nabil AAA, Mohebbi A, Afshar Mogaddam MR (2018) Development of salt and $\mathrm{pH}-$ induced solidified floating organic droplets homogeneous liquid-liquid microextraction for extraction of ten pyrethroid insecticides in fresh fruits and fruit juices followed by gas chromatography-mass spectrometry. Talanta 176:565-572

27. Jouyban A, Farajzadeh MA, Afshar Mogaddam MR (2020) In matrix formation of deep eutectic solvent used in liquid phase extraction coupled with solidification of organic droplets dispersive liquid-liquid microextraction; application in determination of some pesticides in milk samples. Talanta 206:120169

28. Jing X, He J, Zhao W, Huang X, Wang X (2020) Effervescent tablet-assisted switchable hydrophilicity solvent-based microextraction with solidification of floating organic droplets for HPLC determination of phenolic endocrine disrupting chemicals in bottled beverages. Microchem J 155:104680

29. Farajzadeh MA, Bamorowat M, Mogaddam MRA (2016) Development of a dispersive liquid-liquid microextraction method based on solidification of a floating ionic liquid for extraction of carbamate pesticides from fruit juice and vegetable samples. RSC Adv 114:112939-112948

30. Jing $X$, Yang $L$, Zhao W, Wang F, Chen Z, Ma L, Jia L, Wang $X$ (2019) Evaporation-assisted dispersive liquid-liquid microextraction based on the solidification of floating organic droplets for the determination of triazole fungicides in water samples by high-performance liquid chromatography. J Chromatogr $A$ 1597:46-53

31. Cai $Q$, Zhao T, Zhang $L$, Zhao $P$, Zhu Y, Xu H, Hou X (2019) A new strategy for extraction and depuration of pantoprazole in rat plasma: vortex assisted dispersive micro-solid-phase extraction employing metal organic framework MIL-101(Cr) as sorbent followed by dispersive liquid-liquid microextraction based on solidification of a floating organic droplet. J Pharm Biomed Anal 172:86-93

32. Xue J, Wang RQ, Chen X, Hu S, Bai XH (2019) Three phase hollow-fiber liquid-phase microextraction based on deep eutectic solvent as acceptor phase for extraction and preconcentration of main active compounds in a traditional Chinese medicinal formula. J Sep Sci 42:2239-2246

Publisher's Note Springer Nature remains neutral with regard to jurisdictional claims in published maps and institutional affiliations. 